\title{
CORRESPONDENCE
}

\section{Adult congenital heart disease and pulmonary hypertension: management of a complex case}

\section{To the Editor:}

Patients with congenital heart disease (CHD) are at increased risk of developing pulmonary arterial hypertension (PAH). These patients have a particularly poor prognosis if untreated when compared with CHD patients without PAH [1]. This case study demonstrates how a complete work-up and continuous follow-up in expert centres is required to ensure that PAHCHD patients are managed appropriately.

A female patient initially presented at a local community hospital at 3 yrs of age when she had failed to thrive. Echocardiography showed the presence of a moderately sized ventricular septal defect (VSD) and pulmonary stenosis. She underwent surgery for pulmonary stenosis with right ventricle outflow tract (RVOT) augmentation and valvectomy. However, the VSD was left open for reasons that were not specified in the patient's notes. Following this procedure, the patient moved out of the area and was lost to follow-up.

After $16 \mathrm{yrs}$, in 2006 and at the age of $19 \mathrm{yrs}$, she presented to a local cardiologist to enquire whether she could become pregnant. On assessment, she was found to have mild dyspnoea in World Health Organization functional class II/III, oxygen saturation of $91 \%$ on room air, loud P2 heart sounds, II/VI holosystolic murmur at the left sternal border, normal jugular venous pressure and no evidence of oedema. Transthoracic echocardiography (TTE) showed a mildly enlarged right ventricle with normal systolic function, a moderately sized malaligned VSD with left-to-right (systemic-to-pulmonary) shunting, and normal left ventricle size and systolic function. Based on these findings, the patient was referred for immediate VSD closure. At this time, pregnancy was not ruled out. The patient was subsequently referred to an adult $\mathrm{CHD} /$ pulmonary hypertension $(\mathrm{PH})$ expert centre for further evaluation.

At the adult $\mathrm{CHD} / \mathrm{PH}$ evaluation, the patient complained of dyspnoea with hill walking, climbing stairs or when carrying heavy loads, but she had no symptoms during routine daily activity and denied experiencing syncope or chest pain. She reported that her symptoms had not changed over the past few years. The patient had no other relevant medical history, was not taking any medications and had no family history of CHD or PH. On examination, she appeared well and not overtly cyanotic. Her heart rate was 62 beats $\cdot \mathrm{min}^{-1}$, her blood pressure was 112/76 mmHg and her weight was $60 \mathrm{~kg}$. She had palpable $\mathrm{P} 2$ and an increased right ventricular impulse, normal jugular venous pressure, regular rhythm and rate, II/VI holosystolic murmur, normal pulses without upper or lower extremity delay, no evidence of oedema, and lungs clear to auscultation. Mild finger clubbing was also present. Her ECG showed no evidence of extreme right-axis deviation and no right atrial electrical abnormalities. TTE revealed a malaligned moderate size VSD with largely left-to-right shunting, although a degree of right-to-left shunting was present (fig. 1). An enlarged right ventricle with flattened interventricular septum was also evident. Right ventricular systolic pressure (calculated from the tricuspid regurgitation velocity, poor envelope) was 30$40 \mathrm{mmHg}$ and the RVOT gradient was $<10 \mathrm{mmHg}$. Cardiac magnetic resonance imaging demonstrated a right ventricular ejection fraction of $38 \%$, normal pulmonary veins, no evidence of pulmonary artery stenosis, normal aortic size and no additional shunts. On cardiac catheterisation her pulmonaryto-systemic flow ratio (Qp/Qs) was $1.4 / 1$, Qp was $4.2 \mathrm{~L} \cdot \mathrm{min}^{-1}$ and pulmonary vascular resistance (PVR) was 9 Wood units; there was no marked change in these values on vasodilator challenge. Pulmonary function testing showed normal volumes and flows, with a diffusing capacity of the lungs for carbon monoxide of $68 \%$. Exercise testing revealed a 6-min walk distance (6MWD) of $410 \mathrm{~m}$, with a decrease in oxygen saturation from $91 \%$ at rest to $84 \%$ at peak exercise and an increase in Borg dyspnoea index from 0 to 3 . The patient was scheduled for follow-up to discuss her test results and to initiate PAH-specific therapy, but failed to attend the appointment and was once again lost to follow-up.

The patient re-presented 5 yrs later at the age of 24 yrs with worsening dyspnoea on exercise. Her oxygen saturation had decreased to $88 \%$ at rest and to $80 \%$ with exercise, and her 6MWD had decreased to $370 \mathrm{~m}$. Cardiac catheterisation showed that her right atrial pressure had increased from 10 to $14 \mathrm{mmHg}$ and her mean pulmonary artery pressure (PAP) from 50 to $58 \mathrm{mmHg}$. The Qp/Qs was $1.1 / 1$ and PVR had increased to 10.4 Wood units. With a large VSD and right-toleft shunting in the face of elevated PVR, she now received the diagnosis of Eisenmenger's syndrome. In subsequent discussions, the patient was advised against pregnancy. Treatment options were discussed and it was decided to initiate PAHspecific therapy with the endothelin receptor antagonist bosentan. 3 months later she reported improved exercise tolerance and her 6MWD had improved to $400 \mathrm{~m}$ with an increase in peak exercise oxygen saturation to $83 \%$.

This case study raises a number of important issues around the management of adult $\mathrm{CHD} / \mathrm{PH}$ patients. Patients who undergo corrective surgery within the first 9 months of life generally have normal PVR within $1 \mathrm{yr}$ and avoid many of the potential sequelae of $\mathrm{CHD}$, including $\mathrm{PAH}$ [2]. Although surgery could be delayed until later in childhood and PVR may still fall, normal levels may not be achieved [3]. Additionally, repair of an intracardiac lesion in the presence of established PAH may accelerate disease progression and the onset of right ventricular failure [2]. These findings suggest 

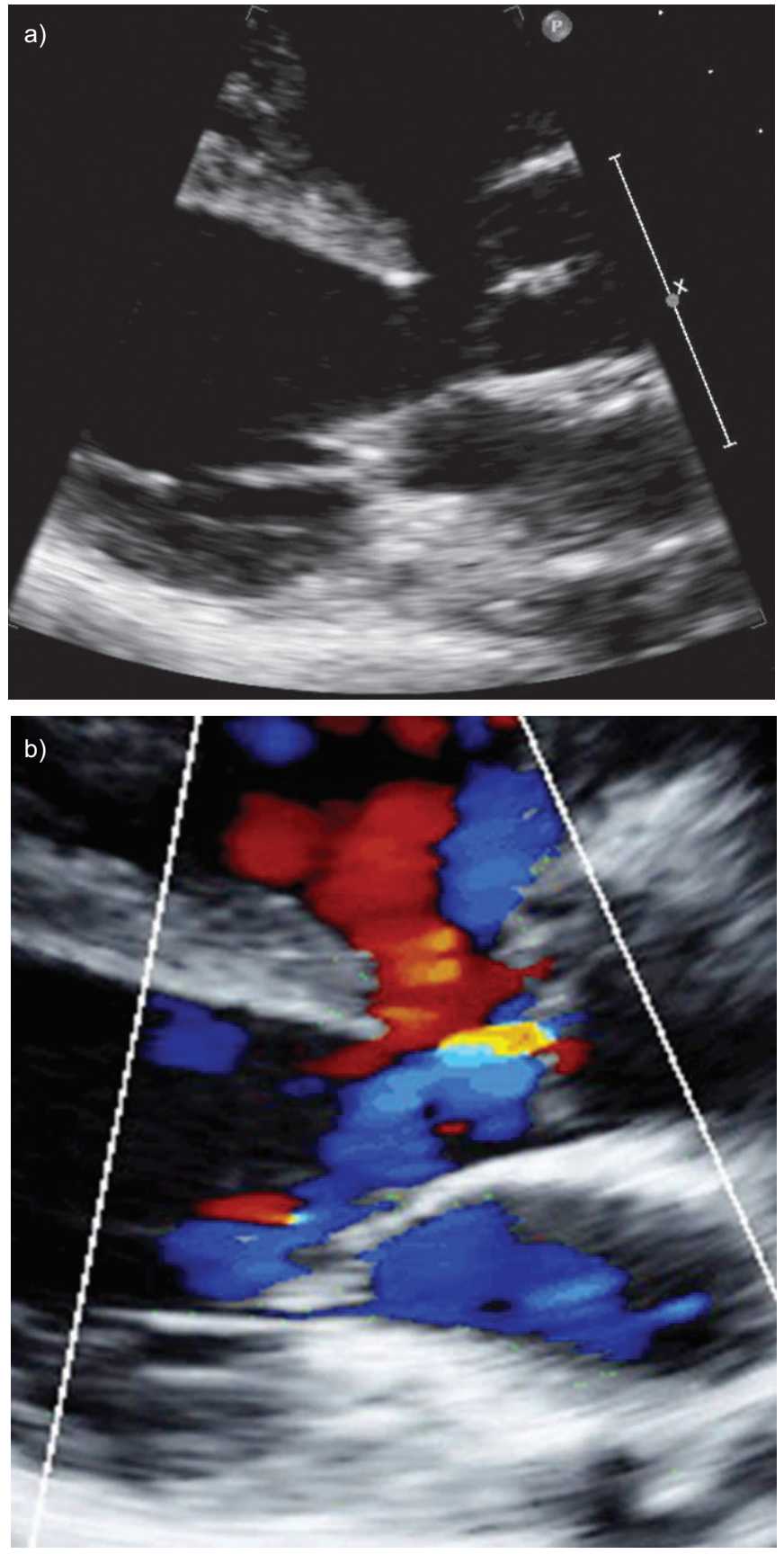

FIGURE 1. a) Transthoracic echocardiography of the patient aged $19 \mathrm{yrs}$ showing a moderately sized malaligned ventricular septal defect with a predominantly left-to-right shunt. b) Enlarged view of a.

that there is a point at which changes to the pulmonary vasculature become irreversible and surgery to repair the underlying defect will not ameliorate the patient's pulmonary disease. However, what the threshold is, and how it can be determined, is currently unclear. In this patient, the reasons for the initial decision not to close the VSD when she first presented at age 3 yrs are unknown.

Current guidelines recommend that adult patients with a VSD and PAH should be considered for surgery when there is a substantial net left-to-right shunt present $(\mathrm{Qp} / \mathrm{Qs}>1.5)$ and there are $\leqslant 4$ Wood units at baseline when challenged with vasodilators [4, 5]. Conversely, a PVR $>8$ Wood units, or a PVR or PAP more than two-thirds of systemic pressures contraindicates closure $[4,5]$. However, this leaves a large group of patients with "borderline" PVR between $\sim 4$ and 8 Wood units in whom the degree of reversibility of PAH is unclear and, therefore, in whom the risk/benefit ratio of surgery is unknown. At her assessment at 19 yrs of age, a Qp/Qs ratio of 1.4/1 and a PVR of 9 Wood units meant that this patient was not considered a candidate for repair. Although there has been interest in the potential to use PAH-specific therapies to reduce PVR prior to surgery, a "treat and close" strategy, there are currently few data to guide this approach and long-term trials are required [6].

One particular problem highlighted in the present case is failure to attend clinic and loss to follow-up. Ensuring regular followup of patients with CHD is important for several reasons: 1) ongoing evaluation of the patient's clinical status; 2) early detection of any deterioration; and 3) initiation of new therapies and treatment strategies as they become available. This patient was first lost to follow-up at an early age, which is not uncommon in this patient population. For example, in a large Canadian study of patients diagnosed with $\mathrm{CHD}$ before the age of 6 yrs, 28\% did not receive cardiac follow-up after their sixth birthday [7]. In the case of this patient, despite re-presenting as an adult and undergoing extensive assessment, she did not return after evaluation at the adult $\mathrm{CHD} / \mathrm{PH}$ centre. During this second period her condition progressed to Eisenmenger's syndrome. In the Dutch CONCOR registry, which included 5,970 adult CHD patients with corrected and uncorrected defects, $\sim 40 \%$ had left-to-right shunts and of these one in 10 ( $4 \%$ of patients overall) developed PAH [8]. Overall, $1 \%$ of the total population included in the registry had Eisenmenger's syndrome [8]. The risk of developing PAH and Eisenmenger's syndrome varies depending on the size and location of the underlying cardiac defect and is higher in patients with large septal defects and, in particular, those with septal defects plus complex lesions [8, 9]. The development of PAH in CHD patients, and particularly the development of Eisenmenger's syndrome, is associated with poorer long-term survival relative to patients with CHD who do not develop PAH [1]. In fact, patients with CHD and an open shunt, as seen in this patient, may benefit from the shunt acting as a "pressure relief valve" for high right ventricle pressures; therefore, maintaining cardiac output. However, this could be at the cost of cyanosis and a plethora of cyanotic complications [10].

Eisenmenger's syndrome is a multisystem disorder and patients require careful management. In this case, the patient was considered to be a candidate for PAH-specific therapy. The majority of available data in Eisenmenger's syndrome patients favour the use of the endothelin receptor antagonist bosentan, which has been shown to improve PVR and exercise capacity in without having an adverse effect on systemic oxygen saturations in a double-blind placebo-controlled study [11]. Treatment with advanced therapies (predominantly bosentan) has been shown to significantly improve long-term survival in patients with Eisenmenger's syndrome [12] and to result in long-term maintenance of improvements in 6MWD and oxygen saturation at peak exercise [13]. However, in the study by Dimopoulos et al. [12], approximately one in five 
patients needed escalation of therapy over time, further emphasising the importance of follow-up in these patients.

This patient's initial reason for returning to the clinic at $19 \mathrm{yrs}$ of age was to see if it would be advisable for her to become pregnant. Despite improvements in recent decades, maternal mortality remains very high in patients with $\mathrm{PAH}$, and has been reported to be $28 \%$ in patients with PAH-CHD [14]. Maternal CHD is associated with a markedly increased risk of adverse cardiovascular events (particularly arrhythmia), death during early stages of labour [15] and complications during delivery [16]. These events are a particular concern in females with open VSDs [16]. The combination of severe PAH and cyanosis seen in Eisenmenger's syndrome is associated with especially high maternal mortality rates (50\%) [14]. In contrast to patients with idiopathic PAH where the number of deaths are roughly similar during pregnancy and post-partum, almost all deaths in Eisenmenger's syndrome patients occur during the post-partum period [14]. This is possibly a reflection of the greater ability of the "trained" right ventricle, which has been subjected to chronic pressure overload for a considerable period of time, to cope with pregnancy-induced increases in load [14]. Due to these high risks, pregnancy in patients with PAH-CHD is contraindicated in treatment guidelines [17]. Despite this, there is evidence that the number of pregnancies in patients with CHD is increasing at a greater rate than in the general population [15]. There are limited data on $\mathrm{PAH}-$ specific therapies in pregnant females with PAH-CHD. In the systematic review by BÉDARD et al. [14], neither nitric oxide nor prostacyclins appeared to be of benefit, although both drugs were only administered when the patients were already unstable or had signs of refractory heart failure [14]. There is still a question about whether the elective use of PAH-specific therapy at an earlier stage of pregnancy and labour, for those therapies which are not contra-indicated in pregnancy, might be more beneficial; however, this requires investigation.

\section{Curt J. Daniels*,\#}

*Division of Cardiovascular Medicine, The Ohio State University, and \#The Heart Center, Nationwide Children's Hospital, Columbus, OH, USA.

Correspondence: C.J. Daniels, Nationwide Children's Hospital, 700 Children's Drive, Columbus, OH 43205, USA. E-mail: curt.daniels@nationwidechildrens.org

Statement of Interest: C.J. Daniels' institution receives grants from pharmaceutical companies for research projects. C.J. Daniels does not directly receive financial compensation.

Provenance: Publication of this peer-reviewed article was supported by Actelion Pharmaceuticals Ltd, Switzerland (principal sponsor, European Respiratory Review issue 126).
Acknowledgements: I received editorial assistance from L. Quine (Elements Communications Ltd, Westerham, UK) supported by Actelion Pharmaceuticals Ltd (Allschwil, Switzerland).

\section{REFERENCES}

1 Lowe BS, Therrien J, Ionescu-Ittu R, et al. Diagnosis of pulmonary hypertension in the congenital heart disease adult population impact on outcomes. J Am Coll Cardiol 2011; 58: 538-546.

2 Haworth SG. Pulmonary hypertension in the young. Heart 2002; 88: 658-664.

3 Balint $\mathrm{OH}$, Samman A, Haberer K, et al. Outcomes in patients with pulmonary hypertension undergoing percutaneous atrial septal defect closure. Heart 2008; 94: 1189-1193.

4 Baumgartner H, Bonhoeffer P, De Groot NM, et al. ESC Guidelines for the management of grown-up congenital heart disease (new version 2010). Eur Heart J 2010; 31: 2915-2957.

5 Warnes CA, Williams RG, Bashore TM, et al. ACC/AHA 2008 guidelines for the management of adults with congenital heart disease. J Am Coll Cardiol 2008; 52: e143-e263.

6 Beghetti M, Galiè N, Bonnet D. Can "inoperable" congenital heart defects become operable in patients with pulmonary arterial hypertension? Dream or reality? Congenit Heart Dis 2012; 7: 3-11.

7 Mackie AS, Ionescu-Ittu R, Therrien J, et al. Children and adults with congenital heart disease lost to follow-up: who and when? Circulation 2009; 120: 302-309.

8 Duffels MG, Engelfriet PM, Berger RM, et al. Pulmonary arterial hypertension in congenital heart disease: an epidemiologic perspective from a Dutch registry. Int J Cardiol 2007; 120: 198-204.

9 Warnes CA, Liberthson R, Danielson GK, et al. Task force 1: the changing profile of congenital heart disease in adult life. J Am Coll Cardiol 2001; 37: 1170-1175.

10 Diller G-P, Dimopoulos K, Kafka H, et al. Model of chronic adaptation: right ventricular function in Eisenmenger syndrome. Eur Heart J Suppl 2007; 9: Suppl. H, H54-H60.

11 Galiè N, Beghetti M, Gatzoulis MA, et al. Bosentan therapy in patients with Eisenmenger syndrome: a multicenter, double-blind, randomized, placebo-controlled study. Circulation 2006; 114: 48-54.

12 Dimopoulos K, Inuzuka R, Goletto S, et al. Improved survival among patients with Eisenmenger syndrome receiving advanced therapy for pulmonary arterial hypertension. Circulation 2010; 121: 20-25.

13 Diller GP, Alonso-Gonzalez R, Dimopoulos K, et al. Disease targeting therapies in patients with Eisenmenger syndrome: response to treatment and long-term efficiency. Int J Cardiol 2012; [Epub ahead of print DOI: 10.1016/j.jicard.2012.02.007].

14 Bédard E, Dimopoulos K, Gatzoulis MA. Has there been any progress made on pregnancy outcomes among women with pulmonary arterial hypertension? Eur Heart J 2009; 30: 256-265.

15 Opotowsky AR, Siddiqi OK, D'Souza B, et al. Maternal cardiovascular events during childbirth among women with congenital heart disease. Heart 2012; 98: 145-151.

16 Karamlou T, Diggs BS, McCrindle BW, et al. A growing problem: maternal death and peripartum complications are higher in women with grown-up congenital heart disease. Ann Thorac Surg 2011; 92: 2193-2198.

17 Galiè N, Hoeper MM, Humbert M, et al. Guidelines for the diagnosis and treatment of pulmonary hypertension. Eur Respir J 2009; 34: 1219-1263.

DOI: 10.1183/09059180.00004912 